\title{
Classification and prevalence of spin in abstracts of non-randomized studies evaluating an intervention
}

\author{
Clément Lazarus ${ }^{1,2,3}$, Romana Haneef ${ }^{1,2}$, Philippe Ravaud ${ }^{1,2,3,4,5}$ and Isabelle Boutron 1,2,3,4*
}

\begin{abstract}
Background: Spin represents specific reporting strategies, either intentional or unintentional, to convince the reader that the beneficial effect of the experimental intervention in terms of efficacy and safety is greater than that shown by the results. The objectives of this study were to 1) develop a classification of spin specific to non-randomized studies assessing an intervention and 2) estimate the prevalence of spin in abstracts of reports of such studies.
\end{abstract}

Methods: In a first step, we developed a specific classification of spin for non-randomized studies by a literature review and pilot study. In a second step, 2 researchers trained in the field of methodology evaluated the prevalence of spin in the abstract of all non-randomized studies assessing an intervention published in the BioMed Central Medical Series journals between January 1, 2011 and December 31, 2013. All disagreements were resolved by consensus. We also determined whether the level of spin in abstract conclusions was high (spin reported without uncertainty or recommendations for further trials), moderate (spin reported with some uncertainty or recommendations for further trials) or low (spin reported with uncertainty and recommendations for further trials).

Results: Among the 128 assessed articles assessed, 107 (84\%) had at least one example of spin in their abstract. The most prevalent strategy of spin was the use of causal language, identified in 68 (53 \%) abstracts. Other frequent strategies were linguistic spin, inadequate implications for clinical practice, and lack of focus on harm, identified in 33 (26\%), 25 (20\%), and 34 (27\%) abstracts respectively. Abstract conclusions of 61 (48 \%) articles featured a high level of spin.

Conclusion: Abstract of reports of non-randomized studies assessing an intervention frequently includes spin. Efforts to reduce the prevalence of spin in abstract for such studies are needed.

\section{Introduction}

Spin, or distortion of study findings, can be used by authors to enhance their study findings more than the results justify $[1,2]$. Recent studies demonstrated a high prevalence of spin in study reports. Spin was found in more than half of the abstract conclusions of randomized controlled trials with statistically non-significant results for the primary outcome [3]; moreover, one third of reports of diagnostic accuracy studies contained a form of over-interpretation [4]. The spin used consisted

\footnotetext{
* Correspondence: isabelle.boutron@htd.aphp.fr

'METHODS Team, Centre of Research in Epidemiology and Statistics

Sorbonne Paris Cité (CRESS), UMR 1153, INSERM, Paris, France

${ }^{2}$ Paris Descartes University, Sorbonne Paris Cité, Faculté de Médecine de

Paris, Paris, France

Full list of author information is available at the end of the article
}

mainly of a focus on statistically significant results (within-group comparison, secondary outcomes, subgroup analyses, modified population of analyses); or interpreting statistically non significant results for the primary outcomes as showing treatment equivalence or comparable effectiveness. A recent study in the field of cancer found that the prevalence of spin has increased over time [5]. In the same field, a randomized controlled trial demonstrated that spin in abstracts could modify a reader's interpretation of study results [6].

Non-randomized studies are commonly used in medical research to evaluate interventions. They are particularly useful to draw conclusions about the safety or efficacy of interventions in real-world settings, to assess rare or long-term adverse events or when randomization is not possible (e.g., surgical procedures). However, these 
designs have important limitations. Non randomized designs are also susceptible to many type of spin which could be same or different from those previously described [7]. Particularly, contrary to randomized clinical trials, they may not allow for establishing causal inferences but rather, only an association [8-10].

Our study aimed to 1) develop a classification of spin for non-randomized studies assessing therapeutic interventions and 2) estimate the prevalence of spin in the abstracts of non-randomized studies evaluating a therapeutic intervention published in BioMed Central Medical Series journals.

\section{Methods}

\section{Development of a classification of spin}

Spin was defined as the use of specific reporting strategies, either intentional or unintentional, to convince the reader that the beneficial effect of the experimental treatment in terms of efficacy and safety is higher than is actually shown by the data.

To develop the classification of spin, we performed a literature review of studies of spin for other study designs $[3-5,9,11]$ as well as studies of distorted presentation and interpretation of findings from non-randomized studies [12-19]. From these data, we developed a preliminary classification, discussed among the authors and tested by two 2 researchers (CL and $\mathrm{RH}$ ) with a sample of 15 articles. The classification was discussed until consensus was achieved among the authors.

\section{Prevalence of spin in abstracts}

We selected a sample of reports of non-randomized studies evaluating an intervention published in 25 journals of the BioMed Central Medical Series that regularly publish clinical studies. We selected these journals because they are open-access and publish reports of non-randomized studies evaluating therapeutic interventions from a large range of medical specialties.

\section{Search strategy}

We searched MEDLINE via PubMed (search date January $21,2014)$ for all articles published in the 25 BioMed Central Medical Series journals between January 1, 2011 and December 31, 2013. The list of selected journals and complete search strategy are respectively in Additional files 1 and 2 .

\section{Study identification}

One researcher $(\mathrm{CL})$ screened all titles, abstracts and, if necessary, the full-text articles of the citations retrieved and selected all reports of non-randomized studies assessing a therapeutic intervention defined as a pharmacological or non-pharmacological treatment (e.g., pharmaceutical drugs, surgery, therapeutic education, rehabilitation, paramedical care etc.) proposed to patients to improve their health. We excluded medico-economic assessments of therapeutic interventions and protocols of observational studies (Fig. 1). As a quality control, a second trained researcher $(\mathrm{RH})$ assessed a random selection of $10 \%$ of articles retrieved by the bibliographic search to ensure that some articles were not missed. This second researcher did not retrieve any missed articles in this subset. For every included article, we retrieved the full-text article and the abstract.

\section{Data extraction}

For each selected article, 2 researchers (CL and RH) trained in the field of methodology independently read the abstract and full-text article and collected data on the general characteristics of the study-study design, sample size, type of therapeutic intervention, comparator, funding sources and whether registration was recorded-using a standardized data-extraction form. They systematically searched for spin in the abstracts using the classification system developed previously.

\section{Level of spin in abstract conclusions}

The 2 researchers evaluated the level of spin in the abstract conclusions. A low level of spin was defined as spin reported with uncertainty in the framing and recommendations for further trials, a moderate level as spin reported with some uncertainty in the framing or recommendations for further trials, and a high level as spin reported without any uncertainty or recommendations for further trials.

Any discrepancies were solved by consensus and, if needed, by consultation with a third researcher (IB).

\section{Statistical analysis}

Data are reported with median (Q1-Q3) for continuous variables and number (\%) for categorical variables. Statistical analyses involved use of $\mathrm{R} 2.15 .0$ (http://www.R-project.org, the R Foundation for Statistical Computing, Vienna, Austria) and any appropriate packages.

\section{Results}

\section{Selected articles}

The search strategies retrieved 1,734 citations; 128 were selected and assessed. The description of included articles is in Table 1. The study designs of the selected articles were prospective cohort studies ( $\mathrm{n}=42,33 \%)$, historical cohort studies $(\mathrm{n}=39,30 \%)$ and before-after studies $(\mathrm{n}=40,31 \%)$. The interventions evaluated were drugs $(\mathrm{n}=51,40 \%)$, non-pharmacologic interventions (e.g., surgery, device or equipment, behavioral intervention or participative; $n=48,38 \%)$, and therapeutic strategy $(n=29$, $23 \%)$. The median [Q1-Q3] sample size was 130 [51-458]. The funding source was mainly non-profit $(n=54,42 \%)$, 


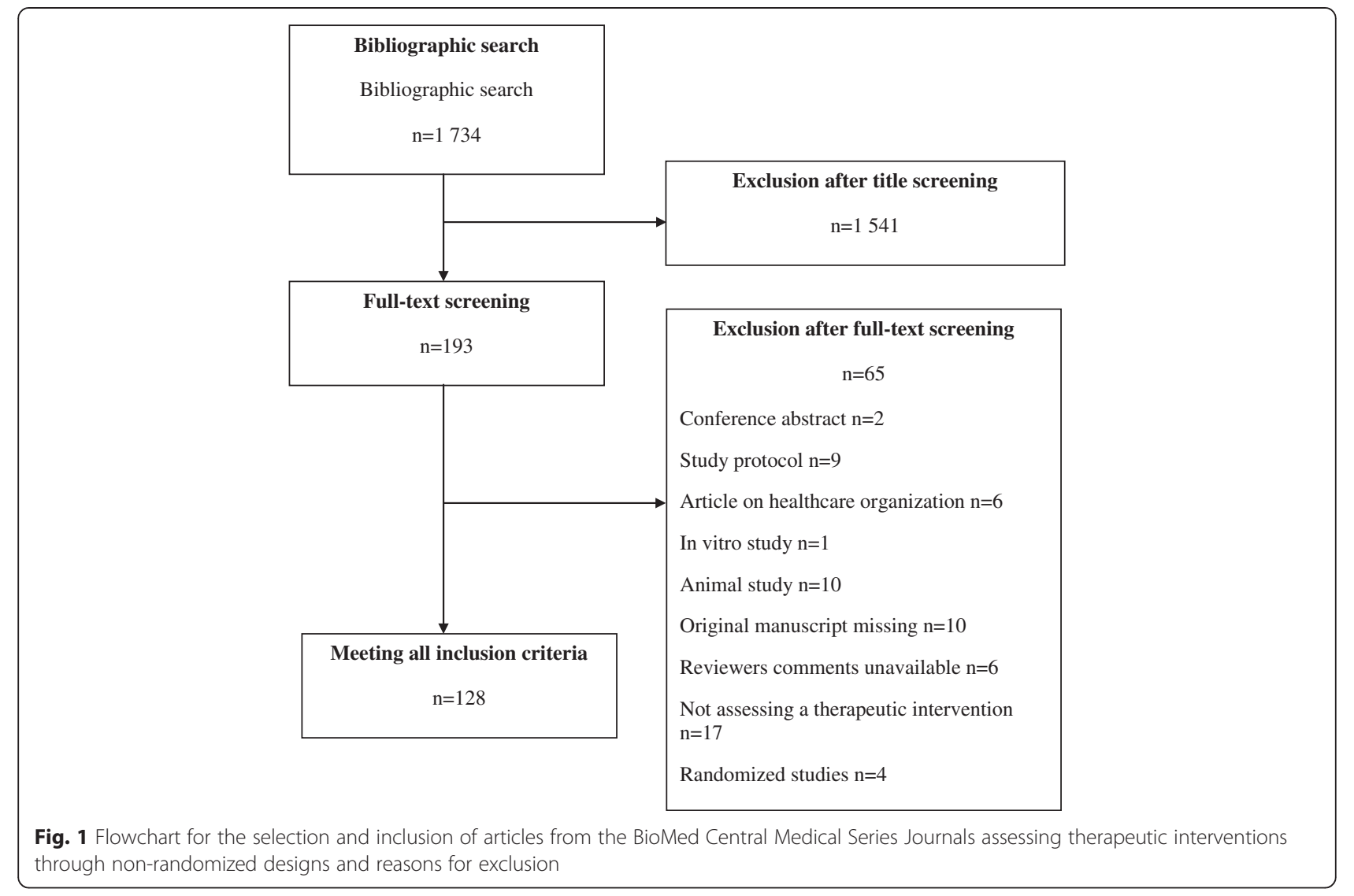

but for 41 articles $(32 \%)$ the funding source was not reported or was unclear.

\section{Classification of spin}

The classification of spin we developed was divided into 3 categories: misleading reporting, misleading interpretation and inadequate extrapolation of the results. Each of these categories included several spin strategies, which are detailed below. Table 2 provides a clear definition of each spin category with an example of spin:

- Misleading reporting of the results was defined as incomplete reporting of the study results that could be misleading for the reader. This type of spin included 1) not reporting adverse events or lack of focus on harms (e.g., no warning on important safety issues), 2) selective reporting of outcomes favoring the beneficial effect of the experimental treatment (e.g., statistically significant results for efficacy outcomes or statistically non-significant results for harm outcomes), 3) misleading reporting of study design, 4) use of linguistic spin or "hype" (i.e., rhetorical manipulations to convince the readers of the beneficial effect of the treatment such as "excellent" results, "encouraging" outcomes, "a trend toward significance"), 5) no consideration of limitations, and 6) selective citation of other studies.

- Inadequate interpretation of the results was defined as misleading interpretation of the study results overestimating the beneficial effect of the intervention. This type of spin included 1) claiming a beneficial effect of the intervention despite statistically non-significant results, 2) claiming an equivalent effect of the interventions for statistically non-significant results despite wide confidence interval, 3) claiming that the treatment is safe for statistically non-significant safety outcomes despite lack of power, 4) concluding a beneficial effect despite no comparison test performed, 5) interpretation of the results according to statistical significance ( $\mathrm{p}$-value) instead of clinical relevance, or 6) claiming a causal effect between the intervention being assessed and the outcome of interest despite a non-randomized design. Use of causal language was defined as any statement addressing the causal relationship of the intervention and outcomes with 1) modal auxiliary verbs, with the intervention as the subject and the outcome as a direct object (e.g., "Surgical experience could shorten the duration of TVT [tension-free vaginal tape] surgery." [20]); 2) use of terms belonging to the semantic field of 
Table 1 Characteristics of included articles

\begin{tabular}{ll}
\hline Characteristics & $n=128(\%)$ \\
\hline Study design & $42(33 \%)$ \\
Prospective & $40(31 \%)$ \\
Before-after & $39(30 \%)$ \\
Historical cohort & $6(5 \%)$ \\
Cross-sectional & $1(1 \%)$ \\
Other & \\
Therapeutic intervention & $51(40 \%)$ \\
Drug & $14(11 \%)$ \\
Surgery & $14(11 \%)$ \\
Device or equipment & $20(16 \%)$ \\
Behavioral intervention or & \\
participative & $29(23 \%)$ \\
Therapeutic strategy & \\
Comparator & $49(70 \%)$ \\
Placebo or attention control & \\
intervention & \\
Active treatment & $54(42 \%)$ \\
Usual care or no treatment & $25(20 \%)$ \\
No comparator or Unclear & $44(34 \%)$ \\
Sample size, median [Q1-Q3], (range) & $129.5[50.75-457.5],(5-238600)$ \\
Funding source & \\
Profit & \\
Not for profit & \\
Both & \\
Not reported or unclear & \\
Registration & \\
Yes & \\
\hline
\end{tabular}

causal relationship (e.g., "effective", "improve", "enhance"); or 3) use of a tone inferring a strong result (e.g., "The results demonstrate" or "This study shows that"). We did not consider that causal language was used when authors stated only a co-occurrence between the intervention and the outcome (e.g., "subjects with symptomatic bipolar disorders who relapse frequently showed improvements in each of these areas after treatment with RLAI [risperidone long-acting injection]" [21]). We did not consider causal language as spin in studies using a propensity score or instrumental variables [22].

- Inadequate extrapolation of the results was defined as an inappropriate generalization of the study result by inadequate 1) extrapolation from the population, interventions or outcome actually assessed in the study to a larger population, different interventions or outcomes, or 2) inadequate implications for clinical practice.

\section{Prevalence of spin in abstracts}

In total, 107 (84 \%) reports had at least one type of spin in their abstracts (Table 3). The median number of type of spin per abstract identified was 2 (Q1-Q3 1-3, range $0-6)$. The most prevalent spin strategy related to the use of causal language identified in $68(53 \%)$ abstracts. For example, in a before-after study including 7 patients, the authors stated "Erythropoietin ... increases the oxygen partial pressure in the brain tissue ... in poor grade SAH [subarachnoid aneurismal hemorrhage] ... patients with severe cerebral vasospasm" [23] and in another prospective study including 22 patients, they stated "[Bilevel positive airway pressure-spontaneous/timed] BiPAP $\mathrm{S} / \mathrm{T}$ with AVAP [average volume assured pressure support] ... facilitates rapid recovery of consciousness when compared to traditional BiPAP $\mathrm{S} / \mathrm{T}$ in patients with chronic obstructive pulmonary disease and hypercapnic encephalopathy" [24].

Other frequent strategies of spin were linguistic spin, inadequate implications for clinical practice and lack of focus on harms, in 33 (26\%), 25 (20\%), and 34 (27\%) abstracts, respectively. For example, we considered linguistic spin frequent when authors indicated that the results were close to significance, despite a p-value > 0.05 . For example, in a prospective study including 662 patients, the authors stated "a tendency towards lower all-cause mortality at 3 months with use of Aspirin + dipyramidol" ( $p=0.12)$ [25] and others used superlatives or "hype" to highlight a beneficial effect of the intervention assessed (e.g., "high potential," "considerably helps," "excellent results"). Inadequate implications for clinical practice occurred mainly when authors extrapolated some recommendations for clinical practice from their results (e.g., in a retrospective study of 42 patients: "it is a suitable therapeutic option not only for initial drainage but also for salvage therapy" [26]).

We identified selective reporting in more than $12 \%$ of abstracts, (e.g., in a before-after study of 23 patients: "[Internet-based cognitive behavior therapy] ICBT... with therapist support reduces [obsessive-compulsive disorder] OCD symptoms, depressive symptoms and improves general functioning" [27], with no report of the lack of improvement in quality of life found in this study). Also, in $13 \%$ of abstracts, authors concluded on the safety of the intervention solely by a statistically non-significant difference in safety outcome despite lack of power. For example, in a historical cohort of 54 patients, the authors reported "Intravenous sodium valproate is as effective as intravenous phenytoin as the first-line treatment in status epilepticus ... with no significant cardiovascular compromise" [28], despite more than twice as many deaths in the intravenous penytoin group (30\% vs $11 \%$ ) although not statistically significant. 
Table 2 Spin classification for non-randomized studies assessing therapeutic interventions. Examples provided are from manuscripts and published abstract and full-texts from our sample

\begin{tabular}{lll}
\hline $\begin{array}{l}\text { Category of } \\
\text { spin }\end{array}$ & Strategy used $\quad$ Definition & Example
\end{tabular}

spin

Misleading Not reporting adverse Results are reported without warnings on reporting events or lack of focus on important or relevant safety issue. harm

Selective reporting

Only a subset of the original outcomes or analysis planned in a study is fully reported.

Misleading description of Study design is presented as more robust study design than it is actually.

Use of linguistic spin

Any word or expression emphasizing the beneficial effect of the therapeutic intervention

No consideration of the limitations

Important limitations are not taken into account in the interpretation of the results.

Selective citation of other studies

Inadequate Claim an effect for

interpretation non-statistically significant results

Claim an equivalence for non-statistically significant results despite a wide confidence interval
Ruling out safety for nonstatistically significant results

Causal language or causal claim

Claim of any significant difference despite lack of statistical test

Focus on statistical significance instead of clinical relevance
Therapeutic intervention and comparator are presented as equivalent when a comparison test is not statistically significant with a large confidence interval.

Only previous studies concordant with the current study findings are acknowledged or other important studies in the field are not reported.

Therapeutic intervention is presented as effective despite a non-statistically significant result.

Therapeutic intervention is presented as safe based on a non-statistically significant comparison test, despite a large confidence interval.

\section{Results are presented with a sentence} implying a cause-and-effect link between the intervention and the outcome

Therapeutic intervention and comparator are compared despite no proper statistical test reported.

Results are presented by their statistical significance without considering the clinical relevance of the effect size.
"This trial showed that overnight switch from oral ropinirole to transdermal rotigotine, with a dose conversion ratio of 1.5:1, was well tolerated in Korean patients with no apparent loss of efficacy." 45/116 experienced adverse events and 13 stopped treatments because of adverse events.

"The results suggest that [Internet-based cognitive therapy] ICBT with therapist support has the potential to reduce [obsessive-compulsive disorders] OCD symptoms, depressive symptoms and general functioning". No report of the lack of improvement in quality of life.

"Based on this prospective case control study tranexamic acid seems not to have a benefit in posterior lumbar spine surgery." It was a retrospective study involving 97 patients and nothing was prospective in this study.

"Both operative methods could be performed safely in the early stages after the introduction of surgery and could consistently obtain excellent surgical performance."

"The TABADO program, targeting teenagers in vocational schools, was effective in producing a higher 12-month abstinence rate among all smokers in the intervention group." No adjustment was made on major confounding variables.

"It would be interesting to know its efficacy and safety in correcting high myopic astigmatism and how it changes the shape of the cornea." Several publications already exist in this field.

"The use of [Automated CardioPulmonary Resuscitation] A-CPR resulted in a higher rate of survival to hospital compared with [Conventional CardioPulmonary Resuscitation] CPR" in a restrospective study involving 66 patients where the propensity score adjusted Odds Ratio was 1.69 [0.79; 3.63].

"The authors concluded that: 1) mortality during follow-up was statistically similar for both groups; (...)" In this retrospective cohort study involving 352 patients, the survival rate at 20 year was $69.3 \%$ with mechanical mitral valve substitutes and $56.6 \%$ with biological substitutes. The hazard ratio was not statistically significant but had a wide $95 \%$ confidence interval $(\mathrm{HR}=1.21[0.79 ; 1.86], p=0.386)$.

"Long-term treatment with esomeprazole (20 mg once daily) is well tolerated and efficacious" despite $16.29 \%$ of patients $(n=22)$ experiencing adverse events judged to be possibly related to treatment with esomeprazole (mostly mild and transient).

"Treatment with oral valacyclovir as the sole antiviral therapy resulted in complete resolution of retinitis." This was a before-after study involving 10 patients.

" $\mathrm{pH}$ and $\mathrm{HCO}^{3-}$ significantly increased after hemodialysis sessions with both aspirin only and aspirin + dipyramidol, the increase of $\mathrm{pH}$ and $\mathrm{HCO}^{3-}$ with aspirin only was significantly larger than aspirin + dipyramidol." No statistical test was reported.

"While the [Clinical Global Impression-Schizophrenia] CGI-SCH overall score improved in both groups after switching, there was a significantly greater change in those who switched from olanzapine (difference of 0.29 points, $p=0.013$ )". The CGI-SCH scale range from 0 to 7 . 
Table 2 Spin classification for non-randomized studies assessing therapeutic interventions. Examples provided are from manuscripts and published abstract and full-texts from our sample (Continued)

\begin{tabular}{|c|c|c|c|}
\hline \multirow[t]{2}{*}{$\begin{array}{l}\text { Inadequate } \\
\text { extrapolation }\end{array}$} & $\begin{array}{l}\text { Inadequate extrapolation } \\
\text { to larger population, } \\
\text { intervention or outcome }\end{array}$ & $\begin{array}{l}\text { Results are generalized to another population, } \\
\text { intervention or outcome than those of the } \\
\text { study (such as surrogate outcomes) }\end{array}$ & $\begin{array}{l}\text { "This intervention approach has the potential to impact } \\
\text { on the progression of colorectal cancers and other } \\
\text { cancers or chronic diseases." The intervention focused on } \\
\text { colorectal cancers only. }\end{array}$ \\
\hline & $\begin{array}{l}\text { Inadequate implication } \\
\text { for clinical practice }\end{array}$ & $\begin{array}{l}\text { Authors recommend the use of the } \\
\text { therapeutic intervention for clinical practice. }\end{array}$ & $\begin{array}{l}\text { "One should not hesitate to perform an osteotomy in } \\
\text { difficult cases." in a prospective cohort study involving } \\
109 \text { patients. }\end{array}$ \\
\hline
\end{tabular}

\section{Level of spin in abstract conclusions}

We classified 61 articles (48 \%) as containing a high level of spin in abstract conclusions, 24 (19 \%) a moderate level of spin and 17 (13\%) a low level of spin. Only 26 articles $(20 \%)$ did not have spin in their abstract conclusions.

\section{Discussion}

To our knowledge, this is the first study to develop and use a classification of spin for non-randomized studies. Of the 128 reports we evaluated, $84 \%$ of abstracts

Table 3 Spin in the abstracts of published articles

\begin{tabular}{|c|c|}
\hline \multirow[t]{2}{*}{ Spin categories } & $\begin{array}{l}\text { Presence of at least one } \\
\text { example of spin in the abstract }\end{array}$ \\
\hline & $n=128$ \\
\hline At least one spin in the abstract & $107(84.0)$ \\
\hline \multicolumn{2}{|l|}{ Misleading reporting } \\
\hline $\begin{array}{l}\text { Not reporting adverse events or } \\
\text { lack of focus on harm }\end{array}$ & 34 (26.6) \\
\hline Selective reporting & $15(11.8)$ \\
\hline $\begin{array}{l}\text { Misleading description of study } \\
\text { designs }\end{array}$ & $1(0.8)$ \\
\hline Use of linguistic spin or "hype" & $33(25.8)$ \\
\hline \multicolumn{2}{|l|}{ Inadequate interpretation } \\
\hline $\begin{array}{l}\text { Claim an effect for non-statistically } \\
\text { significant results }\end{array}$ & $7(5.5)$ \\
\hline $\begin{array}{l}\text { Claim equivalence for non- } \\
\text { statistically significant results }\end{array}$ & $13(10.2)$ \\
\hline $\begin{array}{l}\text { Ruling out safety when results are } \\
\text { not statistically significant }\end{array}$ & $15(11.8)$ \\
\hline Causal language or causal claim & $68(53.1)$ \\
\hline $\begin{array}{l}\text { Claim any difference despite no } \\
\text { comparison test performed }\end{array}$ & $1(0.8)$ \\
\hline $\begin{array}{l}\text { Focus on statistical significance } \\
\text { instead of clinical relevance }\end{array}$ & $1(0.8)$ \\
\hline \multicolumn{2}{|l|}{ Inadequate extrapolation } \\
\hline $\begin{array}{l}\text { Inadequate extrapolation to larger } \\
\text { population, intervention or } \\
\text { outcome }\end{array}$ & $11(8.6)$ \\
\hline $\begin{array}{l}\text { Inadequate implications for clinical } \\
\text { practice }\end{array}$ & $25(19.5)$ \\
\hline Other & $5(3.9)$ \\
\hline
\end{tabular}

Data are no. (\%) contained at least one type of spin and $48 \%$ featured a high level of spin. Use of causal language was the most frequent spin strategy. Yet, the use of causal claims is misleading in the interpretation of non-randomized studies because these designs are unable to control for every confounding factor.

Our findings are consistent with results of other studies. Many of the spin strategies we observed are shared with those identified in randomized controlled trials. Spin strategies are varied and frequent and might lead to misleading interpretation of the study results. This situation is problematic because it has been demonstrated [6] that spin in the abstract conclusions of randomized controlled trial reports could bias the interpretation of the results by clinicians [29].

A particular feature of our work was the important prevalence of causal claims in abstracts of nonrandomized studies. Causal language is a specific spin strategy for non-randomized studies. Such designs, as opposed to randomized studies, do not allow for concluding a cause-and-effect link between the assessed intervention and the observed outcome but rather, provide information only about association [8, 30, 31]. Some studies have explored the causal language in such designs. Cofield et al. [30] observed a $31 \%$ rate of causal language in a series of 525 peer-reviewed papers on obesity and nutrition. Brown et al. [32] evidenced a $26 \%$ to $50 \%$ rate of studies ascribing greater inferential strength than the study design warranted. Causal claims in epidemiology are a cornerstone of the interpretation of results [33], especially for complex interventions for which randomization is difficult or impossible [11]. In 2012, journal editors of the HEART Group published an editorial review about "the importance of matching language to type of evidence" [34] and concluded with a plea to investigators and editors to "carefully select language used during reporting to match the type of study conducted." The rating of causal inference by researchers was assessed in a study of 38 randomized clinical trials and 35 non-randomized clinical trials [35]. The results highlighted that authors "might have overstated the strength of causal inference in the abstracts of nonrandomized clinical trials, but appeared to report causality appropriately in the main text." 
Our study has several limitations. First, our sample is not representative of all non-randomized studies assessing therapeutic interventions indexed in PubMed. We choose the BioMed Central series of medical journals because it is an open-access and open peer-review collection involving a wide variety of medical specialties, therapeutic interventions and study designs. These journals are also strongly involved in the requirement of reporting guidelines (STROBE, CONSORT) and transparency policies (ICMJE) from authors. Consequently, we cannot extrapolate our results to other journals. Second, the assessment of spin strategies is subjective because the interpretation of the results highly depends on the context. To address this issue, 2 trained researchers independently collected the data using a standardized form, with discrepancies resolved by consensus and the involvement of a third researcher if necessary. Third, our study could not determine whether the spin strategies were conscious attempts to show the treatment as more beneficial than it actually was. We did not assess the impact of spin in abstracts of non-randomized studies on the interpretation of such studies, which can differ depending on the category of spin considered. It is important to recognize that an abstract may contain some spin item but actually be balanced overall. We attempted to take into account this issue and assessed the level of spin in the abstracts conclusions provided some elements about the importance of uncertainty and call for further research for the global tone of a conclusion.

Further studies should be conducted to determine the impact of spin on the readers' interpretation of the study results. It is possible that the presence of spin item could be counterbalanced by other element in the reporting of abstracts.

\section{Conclusion}

We found a high prevalence of spin in abstracts of reports of non-randomized studies. Misleading interpretation of results of such studies could lead to inadequate clinical practices and erroneous beliefs in the effects of therapeutic interventions. The classification we developed should facilitate efforts to reduce the prevalence of spin.

\section{Additional files}

Additional file 1: Selected journal from the BioMed Central medical journals series. (PDF $7 \mathrm{~kb}$ )

Additional file 2: Complete bibliographic request. (PDF $10 \mathrm{~kb}$ )

\section{Abbreviations}

(Q1-Q3): Interquartile Range; A-CPR: Automated CardioPulmonary Resuscitation; AVAPS: Average Volume Assured Pressure Support; BiPAP S/ T: Bi-level Positive Airway Pressure-Spontaneous / Timed; CGI-SCH: Clinical Global Impression-Schizophrenia; CPR: CardioPulmonary Resuscitation; HR: Hazard Ratio; ICBT: Internet-based Cognitive Behavior Therapy; OCD: Obsessive-Compulsive Disorder; RLAl: Risperidone Long-Acting Injection; SAH: Sub-Arachnoïdal Hemorrhage; TVT: Tension-free Vaginal Tape.

\section{Competing interests}

The authors declare that they have no competing interests.

\section{Authors' contributions}

$\mathrm{CL}$ participated in the study concept and design, acquisition of data, analysis and interpretation of the data, and drafting of the manuscript. $\mathrm{RH}$ participated in acquisition of data. PR participated in the study concept and design, analysis and interpretation of the data, and the critical revision of the manuscript. IB participated in the study concept and design, acquisition of data, analysis and interpretation of the data, drafting of the manuscript and the critical revision of the manuscript. All authors read and approved the final manuscript.

\section{Acknowledgment}

The authors would like to acknowledge Dr Youri Yordanov (funded by Assistance Publique Hôpitaux de Paris (AP-HP) for his critical revision of the manuscript.

This study received no specific funding

$\mathrm{CL}, \mathrm{PR}$, IB were funded by the University Paris Descartes and the Assistance Publique Hôpitaux de Paris (AP-HP)

$\mathrm{RH}$ was funded by the Assistance Publique Hôpitaux de Paris (AP-HP)

\section{Author details}

${ }^{1}$ METHODS Team, Centre of Research in Epidemiology and Statistics Sorbonne Paris Cité (CRESS), UMR 1153, INSERM, Paris, France. ${ }^{2}$ Paris Descartes University, Sorbonne Paris Cité, Faculté de Médecine de Paris, Paris, France. ${ }^{3}$ Centre d'Épidémiologie Clinique, Hôpital Hôtel Dieu, Assistance Publique des Hôpitaux de Paris, 1, place du Parvis Notre-Dame, 75181 Paris, France. ${ }^{4}$ French Cochrane Center, Paris, France. ${ }^{5}$ Department of Epidemiology, Columbia University Mailman School of Public Health, New York, NY, USA.

Received: 21 May 2015 Accepted: 5 October 2015

Published online: 13 October 2015

\section{References}

1. Horton R. The rhetoric of research. BMJ. 1995;310(6985):985-7.

2. Fletcher RH, Black B. "Spin" in scientific writing: scientific mischief and legal jeopardy. Med Law. 2007;26(3):511-25.

3. Boutron I, Dutton S, Ravaud P, Altman DG. Reporting and interpretation of randomized controlled trials with statistically nonsignificant results for primary outcomes. JAMA. 2010;303(20):2058-64.

4. Ochodo EA, De Haan MC, Reitsma JB, Hooft L, Bossuyt PM, Leeflang MM. Overinterpretation and misreporting of diagnostic accuracy studies: evidence of "spin". Radiology. 2013;267(2):581-8.

5. Vera-Badillo FE, Shapiro R, Ocana A, Amir E, Tannock IF. Bias in reporting of end points of efficacy and toxicity in randomized, clinical trials for women with breast cancer. Ann Oncol. 2013;24(5):1238-44.

6. Boutron I, Altman DG, Hopewell S, Vera-Badillo F, Tannock I, Ravaud P: Impact of spin in the abstract of articles reporting results of Randomised Controlled Trials in the field of cancer, the SPIIN Randomised Controlled Trial. Journal of Clinical Oncology 2014 (in press).

7. Dahabreh IJ, Kent DM. Can the learning health care system be educated with observational data? JAMA. 2014;312(2):129-30.

8. Parascandola M, Weed DL. Causation in epidemiology. J Epidemiol Community Health. 2001;55(12):905-12.

9. Vandenbroucke JP. Why do the results of randomised and observational studies differ? BMJ. 2011;343:d7020. doi:10.1136/bmj.d7020.

10. Vandenbroucke JP. When are observational studies as credible as randomised trials? Lancet. 2004;363(9422):1728-31.

11. Rickles D. Causality in complex interventions. Med Health Care Philos. 2009;12(1):77-90.

12. Mathieu S, Boutron I, Moher D, Altman DG, Ravaud P. Comparison of registered and published primary outcomes in randomized controlled trials. JAMA. 2009;302(9):977-84.

13. Bland JM, Altman DG. Comparisons against baseline within randomised groups are often used and can be highly misleading. Trials. 2011;12(264):1745-6215.

14. Hernandez AV, Pasupuleti V, Deshpande A, Thota P, Collins JA, Vidal JE. Deficient reporting and interpretation of non-inferiority randomized clinical trials in HIV patients: a systematic review. PLoS One. 2013;8:5. 
15. Al-Marzouki S, Roberts I, Marshall T, Evans S. The effect of scientific misconduct on the results of clinical trials: a Delphi survey. Contemp Clin Trials. 2005;26(3):331-7.

16. Chan KB, Man-Son-Hing M, Molnar FJ, Laupacis A. How well is the clinical importance of study results reported? An assessment of randomized controlled trials. CMAJ. 2001;165(9):1197-202.

17. Chan AW, Altman DG. Identifying outcome reporting bias in randomised trials on PubMed: review of publications and survey of authors. BMJ. 2005;330(7494):28

18. Hewitt CE, Mitchell N, Torgerson DJ. Listen to the data when results are not significant. BMJ. 2008;336(7634):23-5.

19. Linden $A$. Identifying spin in health management evaluations. J Eval Clin Pract. 2011;17(6):1223-30

20. Ito H, Yamanaka H, Hagiwara M, Furuuchi T, Matsumoto K, Kanai K, et al. Efficacy of tension-free vaginal tape compared with transobturator tape in the treatment of stress urinary incontinence in women: analysis of learning curve, perioperative changes of voiding function. BMC Urol. 2011;11(13):1471-2490

21. Macfadden W, Adler CM, Turkoz I, Haskins JT, Turner N, Alphs L. Adjunctive long-acting risperidone in patients with bipolar disorder who relapse frequently and have active mood symptoms. BMC Psychiatry. 2011;11(171):11-171.

22. Dekkers OM. On causation in therapeutic research: observational studies, randomised experiments and instrumental variable analysis. Prev Med. 2011;53(4-5):239-41.

23. Helbok R, Shaker E, Beer R, Chemelli A, Sojer M, Sohm F, et al. High dose erythropoietin increases brain tissue oxygen tension in severe vasospasm after subarachnoid hemorrhage. BMC Neurol. 2012;12(32):1471-2377.

24. Briones Claudett KH, Briones Claudett M, Chung Sang Wong M, Nuques Martinez A, Soto Espinoza R, Montalvo M, et al. Noninvasive mechanical ventilation with average volume assured pressure support (AVAPS) in patients with chronic obstructive pulmonary disease and hypercapnic encephalopathy. BMC Pulm Med. 2013;13(12):1471-2466.

25. Arnarsdottir L, Hjalmarsson C, Bokemark L, Andersson B. Comparative evaluation of treatment with low-dose aspirin plus dipyridamole versus aspirin only in patients with acute ischaemic stroke. BMC Neurol. 2012;12(67):1471-2377.

26. Inatomi O, Bamba S, Shioya M, Mochizuki Y, Ban H, Tsujikawa T, et al. Threaded biliary inside stents are a safe and effective therapeutic option in cases of malignant hilar obstruction. BMC Gastroenterol. 2013;13(31):13-31.

27. Andersson E, Ljotsson B, Hedman E, Kaldo V, Paxling B, Andersson G, et al. Internet-based cognitive behavior therapy for obsessive compulsive disorder: a pilot study. BMC Psychiatry. 2011;11(125):11-125

28. Tiamkao S, Sawanyawisuth K, Chancharoen A. The efficacy of intravenous sodium valproate and phenytoin as the first-line treatment in status epilepticus: a comparison study. BMC Neurol. 2013:13(98):1471-2377.

29. Kaptchuk TJ. Effect of interpretive bias on research evidence. BMJ. 2003;326(7404):1453-5

30. Cofield SS, Corona RV, Allison DB. Use of causal language in observational studies of obesity and nutrition. Obes Facts. 2010;3(6):353-6.

31. Shahar E, Shahar DJ. On the causal structure of information bias and confounding bias in randomized trials. J Eval Clin Pract. 2009;15(6):1214-6.

32. Brown AW, Bohan Brown MM, Allison DB. Belief beyond the evidence: using the proposed effect of breakfast on obesity to show 2 practices that distort scientific evidence. Am J Clin Nutr. 2013;98(5):1298-308.

33. Blettner M, Heuer C, Razum O. Critical reading of epidemiological papers A guide. Eur J Public Health. 2001;11(1):97-101.

34. Kohli P, Cannon CP. The importance of matching language to type of evidence: avoiding the pitfalls of reporting outcomes data. Clin Cardiol. 2012;35(12):714-7. doi:10.1002/clc.22066. Epub 2012 Nov 28.

35. Li LC, Moja L, Romero A, Sayre EC, Grimshaw JM. Nonrandomized quality improvement intervention trials might overstate the strength of causal inference of their findings. J Clin Epidemiol. 2009;62(9):959-66.

\section{Submit your next manuscript to BioMed Central and take full advantage of:}

- Convenient online submission

- Thorough peer review

- No space constraints or color figure charges

- Immediate publication on acceptance

- Inclusion in PubMed, CAS, Scopus and Google Scholar

- Research which is freely available for redistribution

Submit your manuscript at www.biomedcentral.com/submit 